\title{
A IMPORTÂNCIA DAS VARIAÇÕES ESPECTRAIS TRANSFERIDAS ANATOMICAMENTE PARA A LOCALIZAÇÃO DE FONTES SONORAS
}

\section{Head related transfer function relevance to sound source localization}

\author{
Aline Cabral de Oliveira (1), Leônidas da Silva Morais Netto ${ }^{(2)}$, Luciana Pinto Rocha ${ }^{(3)}$, \\ Vivian Passos Lima ${ }^{(4)}$, Pedro de Lemos Menezes ${ }^{(5)}$
}

\begin{abstract}
RESUMO
Objetivos: investigar a importância das dimensões anatômicas dos ombros e da cabeça para a localização de fontes sonoras por ouvintes normais, em ambiente reverberante; correlacionar o percentual de acertos na localização sonora com o comprimento e largura da cabeça e o comprimento e largura do ombro e observar a diferença entre as diversas freqüências sonoras apresentadas. Métodos: a amostra foi composta por 52 indivíduos, 20 homens e 32 mulheres, os quais foram submetidos aos testes de otoscopia, audiometria tonal liminar, imitanciometria e emissões otoacústicas. Todos apresentaram audição dentro dos padrões de normalidade. Foram realizadas as medidas de comprimento e largura da cabeça e ombros. Posteriormente, os indivíduos foram submetidos ao teste de localização sonora, nos planos horizontal e vertical, nas freqüências de 500, 2.000 e $4.000 \mathrm{~Hz}$. Resultados: obteve-se correlação significativa $(p<0,05)$ apenas entre a largura dos ombros e os acertos no plano horizontal utilizando-se as freqüências de 2 e $4 \mathrm{kHz}$. Conclusão: o percentual de acertos na localização sonora aumenta com o aumento do comprimento dos ombros nas freqüências de 2 e $4 \mathrm{kHz}$, no plano horizontal.
\end{abstract}

DESCRITORES: Localização de Som; Anatomia; Ombro; Som; Filtros; Estimulação Acústica

\section{INTRODUÇÃO}

O homem é capaz de localizar espacialmente a origem de uma fonte sonora em decorrência das mudanças que ocorrem no espectro do som por ele percebido. Estas mudanças acontecem devido às diferenças de intensidade e tempo interaural, somadas às reflexões e difrações da onda sonora na cabeça, nos ombros e nas orelhas externas. A reflexão é uma propriedade de propagação do som no meio, caracterizada pela incidência da onda sonora em um obstáculo, de alta impedância específica,

(1) Fonoaudióloga; Mestre em Ciências Médicas pela Faculdade de Medicina de Ribeirão Preto da Universidade de São Paulo.

(2) Fonoaudiólogo.

(3) Fonoaudiólogo.

(4) Fonoaudiólogo.

(5) Fonoaudiólogo; Professor Assistente da Disciplina Física Acústica e Psicoacústica da Universidade Estadual de Ciências da Saúde de Alagoas; Mestre em Biofísica pela Universidade Federal de Pernambuco; Doutor em Física aplicada à Medicina pela Universidade de São Paulo. que provoca a não continuidade da onda, fazendoa voltar para a posição inicial ${ }^{1-7}$.

Já a difração é caracterizada como um artifício pelo qual a onda sonora contorna obstáculos, incluindo mudança na direção de propagação. Dependendo do tamanho do obstáculo, algumas ondas podem ser melhores difratadas, levando-se em consideração o comprimento de onda $(\lambda)$ das mesmas. O fenômeno é perceptível quando o comprimento de onda for bem maior que o tamanho do obstáculo, havendo então um desvio a partir do objeto interposto. Sendo assim, a difração é influenciada pela freqüência, e, portanto, os sons agudos (alta freqüência) pouco se difratam, em razão de possuírem pequeno comprimento de onda; enquanto que os sons graves (baixa freqüência) difratam-se mais facilmente, em decorrência de seu maior comprimento de onda ${ }^{1,6,8}$.

Assim, por meio da absorção ou da reflexão, os filtros acústicos proporcionam atenuação em algumas freqüências do som que por eles passa ${ }^{7}$. Para uma localização sonora efetiva, é necessário que ocorra uma filtragem do espectro sonoro pelas 
estruturas anatômicas do corpo humano, o que provoca mudanças no som original, denominadas variações espectrais transferidas anatomicamente (VETA). Estas variações estão relacionadas com a freqüência e a origem da fonte sonora, facilitando assim, sua detecção espacial devido às diferentes filtragens ocasionadas. Dentre as estruturas anatômicas do corpo que realizam variações espectrais no som, tem-se: tronco, ombros, cabeça e orelhas ${ }^{7,9-13}$.

Considerando-se as dimensões das estruturas citadas e a velocidade do som no ar (igual a $348 \mathrm{~m} / \mathrm{s}$ ), pode-se calcular as freqüências que melhor são filtradas por cada estrutura, utilizandose a seguinte fórmula: $f=v / \lambda$. Assim, a cabeça produz uma alteração no campo sonoro influenciando, significativamente, os sinais que atingem as orelhas e os canais auditivos ${ }^{7,9,10}$. Existe uma melhor localização sonora espacial nas freqüências acima de $2 \mathrm{kHz}$, visto que essas freqüências apresentam um comprimento de onda aproximadamente igual a $17,5 \mathrm{~cm}$, que equivale, em média, ao diâmetro da cabeça de adultos, calculado através da fórmula descrita anteriormente ${ }^{14,15}$. Segundo este autor, o padrão de comparação das pressões entre o ouvido direito e o esquerdo é definido como sendo a diferença de intensidade interaural (DII). A localização por DII é melhor quando a freqüência sonora possui ondas com comprimentos inferiores ao diâmetro da cabeça - 17,5 cm $(2.000 \mathrm{~Hz})$ - causando uma diferença de pressão entre as orelhas. Porém, outros autores comprovaram que este tipo de localização é melhor para freqüências superiores a $4 \mathrm{kHz}$, cujo comprimento de onda é aproximadamente duas vezes menor que o diâmetro da cabeça ${ }^{16-20}$.

Desta forma, com relação à cabeça, as variações espectrais transferidas modificam a pressão sonora entre as orelhas, ocasionando melhores resultados para as freqüências acima de $4 \mathrm{kHz}^{9-12}$. O ombro, por sua vez, possui comprimento aproximado de $13 \mathrm{~cm}$ e é formado internamente pela cintura escapular, a qual é constituída pela escápula e clavícula ${ }^{14}$. Este comprimento será mais eficiente na reflexão de ondas sonoras acima de $2,6 \mathrm{kHz}$, isto é para comprimentos menores que $13 \mathrm{~cm}(f=v / \lambda)$.

No que se refere aos planos sonoros espaciais, a localização no plano vertical sagital mediano não pode ser realizada através das diferenças interaurais, visto que, de uma posição espacial qualquer para outra, com um ângulo de elevação diferente, as características temporais e de intensidade são praticamente as mesmas ${ }^{21,22}$. No plano vertical sagital mediano, a detecção da origem da fonte sonora se dá devido às mudanças espectrais causadas pelas reflexões e difrações da onda sonora no tronco, nos ombros e nos acidentes do pavi-
Ihão auditivo ${ }^{1}$. Alguns autores complementam que as VETA ajudam também na localização no plano horizontal ${ }^{23,24}$. Assim, os ombros e a cabeça são exemplos de filtros naturais, os quais participam da fisiologia da audição, alterando as características físicas dos sons que chegam às orelhas ${ }^{8}$. A existência de tais estruturas auxilia na proteção auditiva e na compreensão dos sons complexos ${ }^{13,14}$. Desta forma, este estudo tem como objetivo correlacionar o percentual de acertos na localização sonora com o comprimento e largura da cabeça e dos ombros e observar a diferença entre as diversas freqüências sonoras apresentadas e os planos espaciais estudados.

\section{MÉTODOS}

O protocolo desta pesquisa está baseado na legislação pertinente, Resolução № 196/96, do Conselho Nacional de Saúde, do Ministério da Saúde, para estudos com seres humanos.

Foi realizado o cálculo do tamanho da amostra para médias de população infinita através da expressão $\mathrm{n}=\left[\frac{Z_{\alpha / 2} \cdot \sigma}{E}\right]^{2}$. O desvio padrão utilizado foi de 14,7, calculado a partir de teste piloto. O valor de alfa e a precisão absoluta escolhidos foram 0,05 e 4, respectivamente. Assim, o número de pessoas necessário para essa pesquisa foi de 52 .

Os critérios de inclusão estabelecidos foram: a) limiares auditivos iguais ou inferiores a 15 dBNA, com diferenças entre as orelhas por freqüências iguais ou inferiores a $10 \mathrm{~dB}$; b) timpanogramas tipo $\mathrm{A}$, com pico em $0 \mathrm{daPa}$; c) reflexos estapedianos presentes; d) teste de triagem de processamento auditivo central normal; e) idade entre 18 e 50 anos. Os critérios de exclusão foram: a) exposição a ruído ocupacional ou de lazer; b) cirurgias no ouvido; c) mais de três infecções de ouvido no ano corrente; d) uso de medicação ototóxica; d) casos hereditários de surdez; e) experiência anterior com testes de localização sonora espacial.

Após ampla divulgação na Universidade Estadual de Ciências da Saúde de Alagoas, os voluntários compareceram espontaneamente. Os participantes foram selecionados por meio de um questionário de triagem (Figura 1), e estes preencheram e assinaram um "termo de consentimento livre e esclarecido", e receberam explicações sobre a pesquisa. Antes dos testes de localização sonora, foram realizados: otoscopia, para a inspeção do meato acústico externo e da integridade da membrana timpânica; audiometria tonal liminar (tom puro pulsátil) em cabine acústica nas freqüências de 0,$5 ; 1 ; 2 ; 3 ; 4 ; 6$ e $8 \mathrm{kHz}$; imitanciometria e pesquisa dos reflexos estapedianos; triagem do processa- 


\begin{tabular}{|c|c|c|}
\hline \multicolumn{3}{|l|}{$\begin{array}{l}\text { I - IDENTIFICAÇÃO: } \\
\text { № } \quad:\end{array}$} \\
\hline \multicolumn{3}{|l|}{ DATA: } \\
\hline \multicolumn{3}{|l|}{$\begin{array}{l}\text { IDADE: } \\
\text { SEXO: }\end{array}$} \\
\hline \multicolumn{3}{|l|}{ SEXO: } \\
\hline \multicolumn{3}{|l|}{ 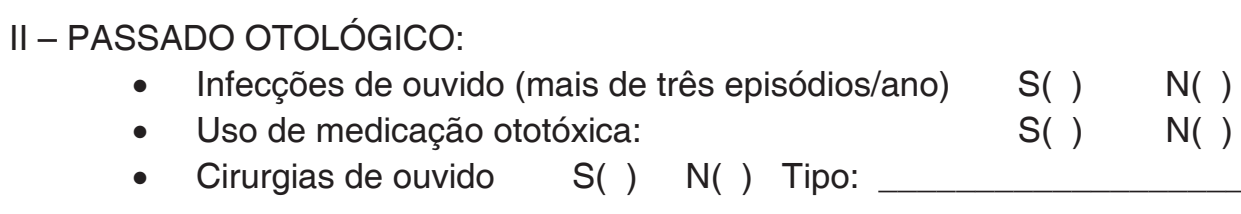 } \\
\hline III - HEREDITARIEDADE PARA SURDEZ: & $S()$ & $N()$ \\
\hline $\begin{array}{l}\text { IV - EXPOSIÇÃO A RUÍDO OCUPACIONAL OU LASER } \\
\text { Em caso afirmativo, Tempo mínimo semanal: }\end{array}$ & $S()$ & $N($ ) \\
\hline V - REPOUSO AUDITIVO MÍNIMO DE 14 HORAS: & $S()$ & $N()$ \\
\hline \multicolumn{3}{|l|}{$\begin{aligned} \text { VI - ESTADO DE SAÚDE GERAL: } \\
$\[ \quad \text { Doenças } \]$ \\
\bullet \quad \text { Medicações }\end{aligned}$} \\
\hline VII - MEATOSCOPIA: & & ente ( ) \\
\hline VIII - TESTES DE IMITANCIOMETRIA: & & nte ( ) \\
\hline IX - AUDIOMETRIA TONAL E VOCAL: & & ente ( ) \\
\hline X - EMISSÕES OTOACÚSTICAS: & & nte ( ) \\
\hline OBS: & & \\
\hline
\end{tabular}

Figura 1 - Questionário aplicado aos sujeitos da pesquisa

mento auditivo central ${ }^{14} \mathrm{e}$ medição das estruturas anatômicas com paquímetro eletrônico digital (comprimento e largura da cabeça e dos ombros). Todos os exames foram realizados em um único dia pela fonoaudióloga pesquisadora.

O teste de localização sonora foi realizado em uma sala reverberante $(3 \times 3 \times 3 \mathrm{~m})$ com níveis ambientais de ruídos de aproximadamente 45dBA e completamente iluminada. Foram utilizados três tipos ondas sonoras quadradas, com freqüências fundamentais de 0,5 , 2 , e $4 \mathrm{kHz}$, escolhidas antecipadamente de forma aleatória.

Para verificação da normalidade amostral foram realizados testes de aderência de KolmogorovSmirnov e de Shapiro-Wilk.
Os dados foram tabulados e processados pelo aplicativo para microcomputador SPSS versão 13.0. Para a descrição dos dados fez-se uso da apresentação tabular e gráfica das médias, dos desvios-padrões e dos percentis.

O protocolo deste estudo foi aprovado pelo Comitê de Ética em Pesquisa da Universidade Estadual de Ciências da Saúde de Alagoas - UNICSAL, com número 255.

Após os dados obtidos serem caracterizados com a utilização de técnicas de estatística descritiva, aplicou-se o teste de regressão linear para correlação entre as variáveis estudadas. Os valores foram considerados significativos para $p$ menor que 0,05 $(p<0,05)$ e o valor do erro beta admitido foi de 0,1 . 


\section{RESULTADOS}

$\mathrm{Na}$ amostra estudada ( $\mathrm{N}=52)$, composta por 20 homens $(38,4 \%)$ e 32 mulheres $(61,6 \%)$, a faixa etária teve média de 21,5 anos e desvio-padrão de 2,09 .

Utilizando-se os testes de Shapiro-Wilk e Kolmogorov-Smirnov foi verificada distribuição normal apenas nas seguintes variáveis: largura e comprimento do ombro, acertos na localização sonora no plano vertical na freqüência de $1 \mathrm{kHz}$ e total de acertos na localização sonora no plano vertical e na freqüência de $2 \mathrm{kHz}$. O que impossibilitou o uso de testes paramétricos.

As médias e desvios padrões das dimensões anatômicas da cabeça e dos ombros, largura e comprimento podem ser analisadas na Tabela 1.

Observando-se apenas os planos espaciais, a média total de acertos na localização sonora foi de $51,01 \%$ e de $35,51 \%$ para os planos espaciais horizontal e vertical, respectivamente. Com relação apenas a freqüência de estimulação, observou-se maior percentual de acertos para a freqüência de $1 \mathrm{kHz}(46,35 \%)$, seguida das freqüências de $2 \mathrm{kHz}$ $(42,17 \%)$ e $4 \mathrm{kHz}(41,27 \%)$.
O percentual médio de acertos por plano espacial e por freqüência pode ser visualizado na Tabela 2, onde se destaca um maior número de acertos no plano horizontal na freqüência de $1 \mathrm{kHz}$.

Aplicando-se o teste de regressão linear para correlacionar os acertos na localização sonora entre as dimensões anatômicas, os planos espaciais e as freqüências de estimulação sonora, obteve-se correlação significativa $(p<0,05)$ apenas entre a largura dos ombros e os acertos no plano horizontal utilizando-se as freqüências de 2 e $4 \mathrm{kHz}$ (Tabela 3). Destaque para a mesma correlação na freqüência de $1 \mathrm{kHz}$, que apesar de não ser significativa, aproximou-se muito $(p=0,06)$.

Em relação ao total de acertos entre os planos e as freqüências de estimulação sonora (Tabela 4), observaram-se valores com significância menor que 0,05 apenas para a largura do ombro no plano horizontal.

O teste de regressão linear revelou ainda que, correlacionando-se o total de acertos em cada freqüência com as dimensões anatômicas dos ombros e da cabeça, não houve valores de $p$ significativos. Entretanto, nas freqüências de 2 e $4 \mathrm{kHz}$, quando considerada a largura do ombro, os valores se aproximaram da significância, Tabela 5 .

Tabela 1 - Dimensões anatômicas médias e desvios padrões, largura e comprimento, da cabeça e dos ombros

\begin{tabular}{lcccc}
\hline & $\begin{array}{c}\text { Largura } \\
\text { da cabeça }\end{array}$ & Comp. cabeça & $\begin{array}{c}\text { Largura } \\
\text { do ombro }\end{array}$ & Comp. do ombro \\
\hline Média & $14,7 \mathrm{~cm}$ & $18,3 \mathrm{~cm}$ & $10,6 \mathrm{~cm}$ & $13,3 \mathrm{~cm}$ \\
Desvio Padrão & $2,2 \mathrm{~cm}$ & $1,7 \mathrm{~cm}$ & $2,1 \mathrm{~cm}$ & $2,5 \mathrm{~cm}$ \\
\hline
\end{tabular}

Tabela 2 - Média de acertos nos planos horizontal e vertical sagital mediano por freqüência

\begin{tabular}{ccc}
\hline $\begin{array}{c}\text { Freqüência } \\
\text { de estimulação }\end{array}$ & Plano horizontal & $\begin{array}{c}\text { Plano vertical sagital } \\
\text { mediano }\end{array}$ \\
\hline $\mathbf{1 ~ k H z}$ & $53,60 \%$ & $39,10 \%$ \\
$\mathbf{2 ~ k H z}$ & $49,60 \%$ & $34,74 \%$ \\
$\mathbf{4 ~ k H z}$ & $49,84 \%$ & $32,70 \%$ \\
\hline
\end{tabular}

\section{DISCUSSÃO}

Neste estudo, pode-se observar menor valor médio para largura da cabeça (igual a $14,7 \mathrm{~cm}$ ) que em estudos anteriores ${ }^{14,15}$, o qual refere que esta medida é de aproximadamente $17,5 \mathrm{~cm}$, entretanto, estas diferenças podem ser justificadas por não se tratar de um estudo realizado na população brasileira. Na literatura, não foram observados valores de normalidade para o comprimento da cabeça e a largura e comprimento dos ombros.

Este estudo revelou que o percentual de acertos na localização sonora foi maior no plano horizontal, em todas as freqüências, corroborando outras pesquisas ${ }^{1,14}$. 
Tabela 3 - Valores de significância ( $p$ ) para correlação de acertos na localização sonora entre as medições anatômicas, os planos espaciais e as freqüências de estimulação sonora

\begin{tabular}{ccccc}
\hline Planos espaciais & Variações anatômicas & $\begin{array}{c}\text { Freqüência } \\
\mathbf{1 ~ k H z}\end{array}$ & $\begin{array}{c}\text { Freqüência } \\
\mathbf{2 ~ k H z}\end{array}$ & $\begin{array}{c}\text { Freqüência } \\
\mathbf{4} \mathbf{~ k H z}\end{array}$ \\
\hline \multirow{4}{*}{ Horizontal } & Largura da cabeça & 0,41 & 0,47 & 0,60 \\
& Comp. da cabeça & 0,59 & 082 & 0,82 \\
& Largura do ombro & $\mathbf{0 , 0 6}$ & $\mathbf{0 , 0 4}$ & $\mathbf{0 , 0 4 ^ { * }}$ \\
& Comp. do ombro & 0,22 & 0,54 & 0,447 \\
\multirow{4}{*}{ Vertical } & Largura da cabeça & 0,30 & 0,89 & 0,619 \\
& Comp. da cabeça & 0,35 & 0,60 & 0,386 \\
& Largura do ombro & 0,54 & 0,16 & 0,223 \\
& Comp. do ombro & 0,38 & 0,97 & 0,672 \\
\hline
\end{tabular}

* Teste de regressão linear significativo

Tabela 4 - Valores de significância ( $p$ ) para correlação entre as variáveis anatômicas e o total de acertos na localização sonora nos planos horizontal e vertical sagital mediano (vsm)

\begin{tabular}{ccc}
\hline Variações anatômicas & Plano horizontal & Plano vsm \\
\hline Largura da cabeça & 0,48 & 0,80 \\
Comprimento da cabeça & 0,73 & 0,41 \\
Largura do ombro & $\mathbf{0 , 0 4}$ & 0,24 \\
Comprimento do ombro & 0,37 & 0,67 \\
\hline
\end{tabular}

* Teste de regressão linear significativo

Tabela 5 - Valores de significância ( $p$ ) para correlação entre as variáveis anatômicas e o total de acertos na localização sonora por freqüência de estimulação

\begin{tabular}{cccc}
\hline Variações anatômicas & Total 1 kHz & Total 2 kHz & Total 4 kHz \\
\hline Largura da cabeça & 0,34 & 0,64 & 0,93 \\
Comprimento da cabeça & 0,93 & 0,90 & 0,79 \\
Largura do ombro & 0,17 & $\mathbf{0 , 0 8}$ & $\mathbf{0 , 0 8}^{*}$ \\
Comprimento do ombro & 0,26 & 0,76 & 0,52 \\
\hline
\end{tabular}

* Próximos da significância

No plano vertical sagital mediano, observou-se um maior número de acertos na freqüência fundamental de $1 \mathrm{kHz}$, o que vai de encontro com outro estudo ${ }^{14,15}$, que afirma ocorrer uma melhor localização sonora espacial nas freqüências acima de $2 \mathrm{kHz}$, e com outros autores ${ }^{9,10,16}$ que mencionam existir melhores resultados para as freqüências acima de $4 \mathrm{kHz}$.

O comprimento dos ombros, de fato, interfere na localização de fontes sonoras. Como havia sido previsto anteriormente, esta medida melhoraria a localização para freqüências com comprimento de onda menor, isto é, freqüências mais agudas. Assim, para comprimentos médios, próximos a $11 \mathrm{~cm}$, deveria haver maior eficiência para as freqüências de 2 e $4 \mathrm{kHz}$, exatamente como pôde ser observado ${ }^{9,10,14,15}$. Fato que explica também a proximidade da significância para o valor de $p(0,06)$ na freqüência de $1 \mathrm{kHz}$.

Novos estudos devem ser conduzidos para verificar a influência de outras estruturas anatômicas (concha, helix e os demais acidentes do pavilhão auricular) na localização de fontes sonoras. 


\section{CONCLUSÃO}

O percentual de acertos na localização sonora é melhor no plano horizontal que nos demais planos espaciais. O percentual de acertos na localização sonora aumenta apenas com o aumento do comprimento dos ombros nas freqüências de 2 e $4 \mathrm{kHz}$, no plano horizontal. Não foi observada correlação entre os acertos e as demais medidas antropométricas realizadas.

\begin{abstract}
Purpose: to investigate the relevance of the head transfer function related to the sound localization by human normal hearing, in reverberant environment; to correlate the percentage of success in sound localization and the length and the width of the head and the shoulders, observing the difference among frequencies and the spatial planes. Methods: the sample was made up by 52 individuals, 20 men and 32 women, which had been submitted to otoscopy, tonal audiometry and OAE. The measures related to length and width of the head and shoulders had been carried out too. Later, the individuals had been submitted to sound localization test, in the horizontal and vertical planes, and with the frequencies of $0.5,2$ and $4 \mathrm{kHz}$. Results: significant correlation was obtained $(p<0.05)$ just between the width of the shoulders and the success in the horizontal plane, using 2 and $4 \mathrm{kHz}$. Conclusion: The percentage of success in the sound localization increases with the increase in the length of the shoulders using 2 and $4 \mathrm{kHz}$, in the horizontal plane.
\end{abstract}

KEYWORDS: Sound Localization; Anatomy; Shoulder; Sound; Filters; Acoustic Stimulation

\section{REFERÊNCIAS}

1. Middlebrooks JC, Green DM. Sound localization by human listeners. Ann Rev Psychol. 1991; 42:135-59.

2. Luntz M, Brodsky A, Hafner H, Shpak T, Feiglin $H$, Pratt $H$. Sound localization in patients with cochlear implant: preliminary results. Int $\mathrm{J}$ Pediatr Otorhinolaryngol. 2002; 64(1):1-7.

3. Priwin C, Jönsson R, Hultcrantz M, Granströmb G. BAHA in children and adolescents with unilateral or bilateral conductive hearing loss: a study of outcome. Int J Pediatr Otorhinolaryngol. 2007; 71(1):135-45.

4. Ratelle A, Dufour J, Leroux T, Laroche C, Giguère $C$, Tetu $E$, et al. Travel safety issues related to digital hearing aids: assessment of the effect of compression in sound localization and distance evaluation tasks by blindfolded hearing impaired adults. Int Congress Series. 2005; 1282: 278-82.

5. Subramaniam K, Eikelboom RH, Eager KM, Atlas MD. Unilateral profound hearing loss and the effect on quality of life after cerebellopontine angle surgery. Otolaryngol Head Neck Surg. 2005; 133(3):339-46.

6. Martin RL, McAnally KI. Spectral integration time of the auditory localisation system. Hear Res. 2008; 238:118-23.

7. Menezes PL, Oliveira AC, Moraes L, Rocha LP, Lima VP. Ressonância: um estudo da orelha externa. Pró-Fono. 2004; 16(3):333-40.
8. Zemlin WR. Princípios de anatomia e fisiologia em fonoaudiologia. Porto Alegre: Artmed; 2000. p. 441-60.

9. lida $\mathrm{K}$, Itoh $\mathrm{M}$, Itagaki $\mathrm{A}$, Morimoto M. Median plane localization using a parametric model of the head-related transfer function based on spectral cues. Appl Acoust. 2007; 68:835-50.

10. Nishino $\mathrm{T}$, Inoue $\mathrm{N}$, Takeda $\mathrm{K}$, Itakura $\mathrm{F}$. Estimation of HRTFs on the horizontal plane using physical features. Appl Acoust. 2007; 68(8):897-908.

11. Röttger S, Schröger E, Grube M, Grimm S, Rübsamen R. Mismatch negativity on the cone of confusion. Neuroscie Letters. 2007; 414(2):178-82.

12. Marsalek $P$, Kofranek J. Sound localization at high frequencies and across the frequency range. Neurocomputing. 2004; 58-60:999-1006.

13. Macpherson EA, Middlebrooks JC. Listener weighting of cues for lateral angle: the duplex theory of sound localization revisited. J Acoust Soc Am. 2002; 111(5Pt1):2219-36.

14. Menezes PL, Soares IA, Caldas Neto S, Maciel R, Motta MA. Estudo da localização sonora em ouvintes normais. J Bras Fonoaudiol. 2003; 4(15):109-13.

15. Menezes PL, Soares IA, Caldas Neto S, Maciel R, Motta MA. Localização sonora: uma nova perspectiva de estudo para a audiologia. Fonoaudiol Brasil. 2003; 2(3):28-35. 
16. Witton C, Green GG, Rees A, Henning GB. Monoaural and binaural detection of sinusoidal phase modulation of a $500-\mathrm{Hz}$ tone. J Acoust Soc Am. 2000; 108(4):1826-33.

17. Moore JM, Tollin DJ, Yin TC. Can measures of sound localization acuity be related to the precision of absolute location estimates? Hear Res. 2008; 238(1-2):94-109.

18. Phillips DP. A perceptual architecture for sound lateralization in man. Hear Res. 2008; 238(1-2):124-32.

19. Getzmann S. Spatial discrimination of sound sources in the horizontal plane following an adapter sound. Hear Res. 2004; 191(1-2):14-20.
20. Sabin AT, Macpherson EA, Middlebrooks JC. Human sound localization at near-threshold levels. Hear Res. 2005; 199(1-2):124-34.

21. Wazen JJ, Ghossaini SN, Spitzer JB, Kuller M. Localization by unilateral BAHA users. Otolaryngol Head Neck Surgery. 2005; 132(6):928-32.

22. Lewald J, Getzmann S. Horizontal and vertical effects of eye-position on sound localization. Hear Res. 2006; 213(1-2):99-106.

23. Batteau DW. The role of the pinna in human localization. Proc R Soc London B Biol Sci. 1967; 168(11):158-80.

24. Butler RA. An analysis of the monaural displacement of sound in space. Percept Psychophys. 1987; 41(1):1-7.

RECEBIDO EM: 22/06/2007

ACEITO EM: 28/05/2008

Endereço para correspondência:

Rua Dr Antônio Cansanção, 55 ap. 703

Maceió - AL

CEP: $57035-190$

Tel: (82) 3315-6813

Fax: (82) 3315-6813

E-mail: son@ @otlink.com.br 